\title{
DIRECT NUMERICAL SIMULATION OF SOLIDIFICATION WITH EFFECTS OF DENSITY DIFFERENCE
}

\author{
Vu Van Truong \\ Hanoi University of Science and Technology, Vietnam \\ E-mail: vuvantruong.pfae@gmail.com \\ Received April 15, 2015
}

\begin{abstract}
In this paper, direct numerical simulations are presented for solidification with the effects of density difference between the solid and liquid phases. A front-tracking method is used. The solidification front, i.e. the solid-liquid interface separating solid and liquid, is represented by connected elements that move on a rectangular and stationary grid. The Navier-Stokes equations are solved by a projection method on the entire domain including the solid phase. An indicator function reconstructed from the front information is used to set the velocities in the solid phase to zero, and thus to enforce the no-slip condition at the interface. The method is validated through comparisons with exact solutions for one- and two-dimensional problems. The method is then used to simulate the solidification processes with the effects of volume change due to density difference.
\end{abstract}

Keywords: Direct numerical simulation, front-tracking, solidification, density difference.

\section{INTRODUCTION}

Solidification problems occur in many industrial applications, and have thus received much attention, through experimental, theoretical and simulation investigations [1]. Experiments can be found in [2-5], in which the authors mentioned the effects of volume change on the phase change process. Many phase change materials such as water, silicon (Si), germanium $(\mathrm{Ge}), \ldots$ have density difference between the solid and liquid phases. Accordingly, volume change always appears during the solidification process, and considerably affects the final products of the phase change process [3].

Phase change problems are non-linear due to the presence of a moving interface between the solid and liquid phases associated with latent heat release. Accordingly, they have a limited number of analytical solutions. Most of them apply to idealized and simplified systems. Some of the basic solutions can be found in [6,7].

Concerning simulations, basically, two different approaches have been used for numerical simulations of the phase change processes: (1) fixed-domain formulation and (2)

(C) 2016 Vietnam Academy of Science and Technology 
variable-domain formulation. The fixed-domain approach can be referred to "enthalpyporosity formulation" [8]. In this approach, the total enthalpy, rather than the temperature, is considered as the primary dependent variable in the energy equation. The enthalpy-porosity formulation combined with moving finite element methods has been developed to solve phase change problems [9]. Other popular methods for tracking interface and moving boundaries in the Eulerian frame are the volume of fluid method [10-12], the level set method [13] and the phase field method [14]. Hybrid methods [15-17] between the Lagrangian and Eulerian approaches have also been proposed for phase change problems. In the variable-domain formulation, body-fitted grid methods [18] have been widely used. However, most of the numerical investigations previously mentioned have neglected volume change. In Vietnam, the volume of fluid method has been used for gas-liquid two-phase flows [19] and for porous media [20]. However, to our knowledge, numerical investigations concerning the phase change process with the above-mentioned issues are still lacking. These gaps motivate our present study on direct numerical simulations of solidification. We here use a front-tracking method for multiphase flows $[16,21,22]$ with modification for solidification accompanied by volume change.

\section{MATHEMATICAL FORMULATION AND NUMERICAL METHOD}

Fig. 1 shows a typical solidification problem with an interface separating solid and liquid. The fusion temperature of the material is $T_{m}$. We assume that the liquid is incompressible and Newtonian, and volume change is assumed to occur only at the phase change front. For computational purposes, we treat all phases as one continuum with variable properties such as density $\rho$, viscosity $\mu$, thermal conductivity $k$ and heat capacity $C_{p}$. In terms of this single-field representation, the momentum and thermal energy equations are

$$
\begin{aligned}
& \frac{\partial(\rho \mathbf{u})}{\partial t}+\nabla \cdot(\rho \mathbf{u u})=-\nabla p+\nabla \cdot\left[\mu\left(\nabla \mathbf{u}+\nabla \mathbf{u}^{T}\right)\right]+\rho \mathbf{g} \\
& \frac{\partial}{\partial t}\left(\rho C_{\mathrm{p}} T\right)+\nabla \cdot\left(\rho C_{\mathrm{p}} T \mathbf{u}\right)=\nabla \cdot(k \nabla T)+\int_{f} \dot{q}_{f} \delta\left(\mathbf{x}-\mathbf{x}_{f}\right) d S .
\end{aligned}
$$

Here, $\mathbf{u}$ is the velocity vector, $p$ is the pressure, and $\mathbf{g}$ is the gravitational acceleration. The interface is denoted by $f$. The Dirac delta function $\delta\left(\mathbf{x}-\mathbf{x}_{f}\right)$ is zero everywhere except for a unit impulse at the interface $\mathbf{x}_{f}$. T and the superscript $T$ denote respectively the temperature and the transpose. $S$ indicates the solidification surface. $D / D t$ is the material derivative. $\dot{q}_{f}$ is the heat flux at the solidification interface, given as

$$
\dot{q}_{\mathrm{f}}=\left.k_{\mathrm{s}} \frac{\partial T}{\partial n}\right|_{\mathrm{s}}-\left.k_{1} \frac{\partial T}{\partial n}\right|_{1}=\dot{m} L_{h}=-\rho_{\mathrm{s}} V_{n} L_{h},
$$

where the subscripts $s$ and $l$ represent solid and liquid, respectively. $V_{n}$ is the velocity normal to the solidification front (Fig. 1) and $L_{h}$ is the latent heat. The velocity field can be written as

$$
\mathbf{u}=\mathbf{u}_{s} I+\mathbf{u}_{l}(1-I),
$$




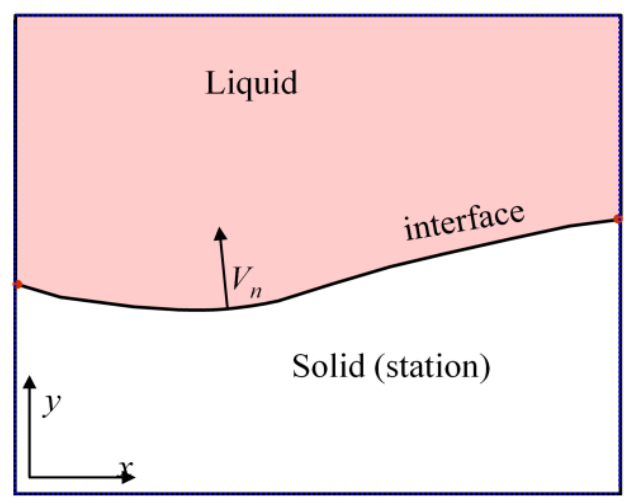

(a) Solidification interface

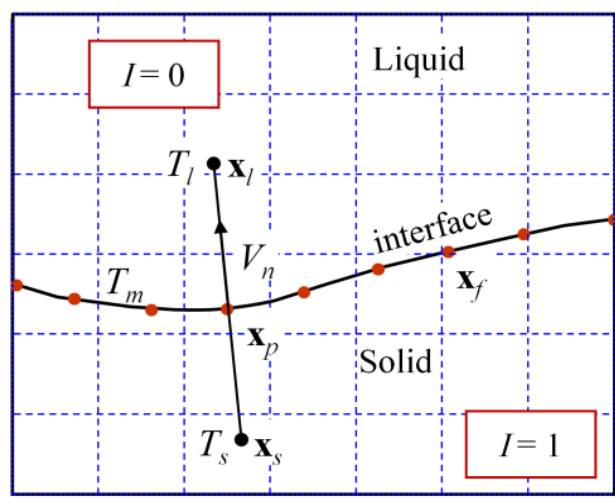

(b) Interface - font tracking representation

Fig. 1. A typical solidification problem with the solidification interface separating liquid and solid

where $I$ is an indicator function which is one in the solid and zero in the fluid (Fig. 1 ). $I$ is determined from front properties

$$
\nabla I=\int_{f} \delta\left(\mathbf{x}-\mathbf{x}_{f}\right) \mathbf{n}_{f} d S,
$$

where $\mathbf{n}_{f}$ is the normal vector to the interface. Mass conservation at the solidification interface gives

$$
\dot{m}=\rho_{l} \mathbf{n}_{f} \cdot\left(\mathbf{u}_{l}-\mathbf{n}_{f} V_{n}\right)=\rho_{s} \mathbf{n}_{f} \cdot\left(\mathbf{u}_{s}-\mathbf{n}_{f} V_{n}\right) .
$$

Using Eq. (5) and taking the divergence of Eq. (4) with $\nabla \cdot \mathbf{u}_{l}=\mathbf{u}_{s}=\mathbf{0}$ yields the following mass conservation equation

$$
\nabla \cdot \mathbf{u}=\int_{\mathrm{f}} \delta\left(\mathbf{x}-\mathbf{x}_{\mathrm{f}}\right)\left(\mathbf{u}_{s}-\mathbf{u}_{l}\right) \cdot \mathbf{n}_{f} d S .
$$

Elimination of $V_{n}$ in Eq. (6) with noting that gives

$$
\left(\mathbf{u}_{l}-\mathbf{u}_{s}\right) \cdot \mathbf{n}_{f}=\frac{\dot{q}_{f}}{L_{\mathrm{h}}}\left(\frac{1}{\rho_{l}}-\frac{1}{\rho_{s}}\right) .
$$

Using Eq. (7) and Eq. (8) yields the following mass conservation

$$
\nabla \cdot \mathbf{u}=\frac{1}{L_{\mathrm{h}}}\left(\frac{1}{\rho_{S}}-\frac{1}{\rho_{l}}\right) \int_{\mathrm{f}} \delta\left(\mathbf{x}-\mathbf{x}_{\mathrm{f}}\right) \dot{q}_{\mathrm{f}} d S .
$$

In summary, the governing equations for the problem includes Eqs. (1), (2) and (9). These equations are solved by the front-tracking/finite difference method on a staggered grid with second order accuracy in time and space. 


\section{NUMERICAL METHOD}

Here we use the front-tracking method [21] with modification accounting for solidification and volume change upon solidification. The phase boundary, i.e. the solidification interface, is represented by line segments that move on the rectangular and fixed grid (Fig. 1). These elements are linked together, and are used to transfer information between the interface and the grid. Movement of the phase boundary points is specified from

where $V_{n}$ is simply given by

$$
D \mathbf{x}_{f} / D t=V_{n} \mathbf{n}_{f}
$$

$$
V_{n}=-\dot{m} / L_{h}=-\dot{q}_{f} /\left(\rho_{s} L_{h}\right) .
$$

The heat source $\dot{q}_{f}$ is calculated using a normal probe technique $[16,22]$ described as follows. At a point $\mathrm{P}\left(\mathbf{x}_{p}\right)$ on the interface we draw a line normal to the interface (Fig. 1b), and specify two points $S$ (in solid) and L (in liquid) on each side of the interface such that where $h$ is the grid spacing. The temperatures $T_{S}$ and $T_{l}$ at these points are interpolated from the nearest grid temperatures $T_{i j}$ by

$$
T_{s, l}=\sum_{i j} T_{i j} D_{i j}\left(\mathbf{x}_{s, l}\right) .
$$

Here, $D_{i j}\left(\mathbf{x}_{s, l}\right)$ is the area weighting function defined as

$$
D_{i j}\left(\mathbf{x}_{s, l}\right)=d\left(x_{s, l} / h-i\right) d\left(y_{s, l} / h-j\right),
$$

where

$$
d(r)=\left\{\begin{array}{l}
1-r, 0<r<1 \\
1+r,-1<r<0 \\
0,|r| \geq 1
\end{array}\right.
$$

$i$ and $j$ are the indices of the fixed grid point, with noting that the indices $i$ and $j$ corresponding with the point $S$ are different from those corresponding with the point $L$.

The heat source at $\mathrm{P}$ is calculated using the first-order finite difference approximation of Eq. (3)

$$
\dot{q}_{p}=\frac{1}{h}\left(k_{s}\left(T_{s}-T_{m}\right)-k_{l}\left(T_{m}-T_{l}\right)\right) .
$$

This heat source is then distributed to the nearest grid points by

$$
Q_{i j}=\sum_{p} \dot{q}_{p} D_{i j}\left(\mathbf{x}_{p}\right) \frac{\Delta s_{p}}{h^{2}},
$$

where $\Delta s_{p}$ is the average of the straight line distances from the point $\mathrm{P}$ to the two points on either side of $\mathrm{P}$ on the interface. Eq. (16) is the discretized form of the last term in Eq. (2), where we have approximated the Dirac function by the area weighting function $D_{i j}$.

The indicator function $I$ is reconstructed from the new position of the interface points. The values of the material properties are then found: $\varphi=\varphi_{\mathrm{s}} I+(1-I) \varphi_{1}$, where $\varphi$ stands for $\rho, \mu \mathrm{s} C_{p}$, or $k$. At this point, we have all information to integrate the energy and Navier-Stokes equations which are descritized using an explicit predictor-corrector 
time-integration method and a second-order centered difference approximation for the spatial derivatives. The discretized equations are solved on a fixed, staggered grid using the MAC method [23]. Since there's the presence of the solid phase, the standard predictor-corrector scheme is modified as follows.

(1) A provisional velocity field $\mathbf{u}^{* *}$ is calculated

$$
\mathbf{u}^{* *}=\left(\Delta t \mathbf{A}^{n}+\rho \mathbf{u}^{n}\right) / \rho^{n+1}
$$

where A represents the right-hand side of Eq. (1) which contains the advection, the diffusion and the gravitational term, and $n$ is the current time.

(2) $\mathbf{u}^{* *}$ is then corrected by $I$

$$
\mathbf{u}^{*}=(1-I) \mathbf{u}^{* *} \text {. }
$$

(3) The pressure is found by solving the Poisson equation

$$
\nabla \frac{1}{\rho^{n+1}} \cdot \nabla p^{n+1}=\frac{\nabla \cdot \mathbf{u}^{*}-\nabla \cdot \mathbf{u}^{n+1}}{\Delta t} .
$$

where $\nabla \cdot \mathbf{u}^{n+1}$ is replaced with the right-hand side of Eq. (9).

(4) The final velocity field at the next time $n+1$ is updated

$$
\mathbf{u}^{n+1}=\Delta t \mathbf{u}^{*}-\Delta t(1-I) \nabla p^{n+1} / \rho^{n+1} .
$$

(5) The temperature field at the next time $n+1$ is found by

$$
T^{n+1}=\left(\Delta t \mathrm{~B}^{n}+\rho^{n} C_{p}^{n}\right) /\left(\rho^{n+1} C_{p}^{n+1}\right),
$$

where B represents the right-hand side of Eq. (2).

\section{RESULTS}

\subsection{One-dimensional problem}

In order validate the method, we first solve a one-dimensional moving boundary problem, and compare the computational results with analytical solutions [6], as shown in Fig. 2. Initially, the liquid is at a uniform temperature $T_{i}$ equal to the melting temperature $T_{m}$. At $t=0$, the bottom boundary at $y=0$ is suddenly lowered to $T_{c}<T_{m}$, and is maintained at this temperature for time $t>0$. As a results, the solidification interface starts at $y=0$, and moves upward. The governing equations of this problem are given, in dimensionless, as

$$
\begin{aligned}
& \partial \theta / \partial \tau=\partial \theta / \partial Y^{2} \text { in } 0<Y<S(\tau), \quad \tau>0 \\
& \theta(S(\tau)<Y \leq 1, \tau>0)=1 \\
& \theta(Y=S(\tau), \tau>0)=1
\end{aligned}
$$

The following dimensionless quantities are used

$$
\theta=\left(T-T_{c}\right) /\left(T_{m}-T_{c}\right), Y=y / H, S=s / H, \tau=\alpha_{l} t / H^{2},
$$




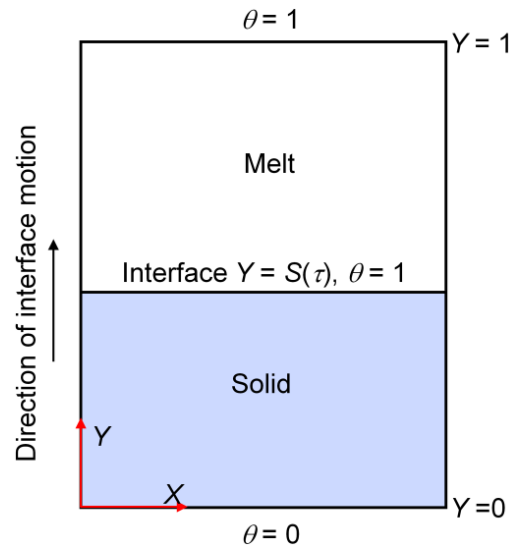

Fig. 2. One-dimensional solidification problem

$\alpha$ is the thermal diffusivity, $s$ is the front location, and $H$ is the domain height. The thermal properties of the solid and liquid are assumed equal. The non-dimensional form of Eq. (3) is

$$
\left.\frac{\partial \theta}{\partial Y}\right|_{\mathrm{s}}-\left.\frac{\partial \theta}{\partial Y}\right|_{1}=\frac{1}{S t} \frac{d S}{d \tau}
$$

where $S t$ is the Stefan number defined as $L_{h}$. The exact solutions of this problem for the temperature and for the front location are respectively given as [6]

$$
\begin{gathered}
\theta=\operatorname{erf}(Y /(2 \sqrt{\tau})) / \operatorname{erf}(\lambda), \\
S=2 \lambda \sqrt{\tau},
\end{gathered}
$$

where $\lambda$ is the root of

$$
\lambda e^{\lambda^{2}} \operatorname{erf}(\lambda)=S t / \sqrt{\pi}
$$

and erf is the error function.

Simulations were performed at two Stefan numbers $S t=0.1$ and 5 , and with a computational domain size of $0.5 \times 1$. The grid resolution is $64 \times 128$. The initial front location and the temperature used for the simulation were found from the exact solutions at the moment $\tau=\tau_{0}$ when a thin solid layer with thickness $S_{0}=0.1$ has formed.

Results for the front location compared with the exact solution and with the computational results reported in [17], for $S t=0.1$ and for $S t=5$ are shown in Fig. 3a and Fig. 3b, respectively. Higher Stefan number corresponds to lower latent heat released, and results in more rapid solidifying. As seen from Fig. 3, agreement between the numerical and exact results is excellent. The average error is less than $0.044 \%$ for $S t=0.1$, and less than $0.06 \%$ for $S t=5$. As compared with the results reported in [17], the present method produces the solutions that are more accurate, as shown in Fig. 3. In addition, as solidification proceeds, the error does not increase. This confirms the reliability and accuracy of the method for resolving the evolution of the front position.

Next, we consider the effect of density difference between the solid and liquid phase, i.e. volume change upon solidification for this 1D problem. Fig. 4 compares 


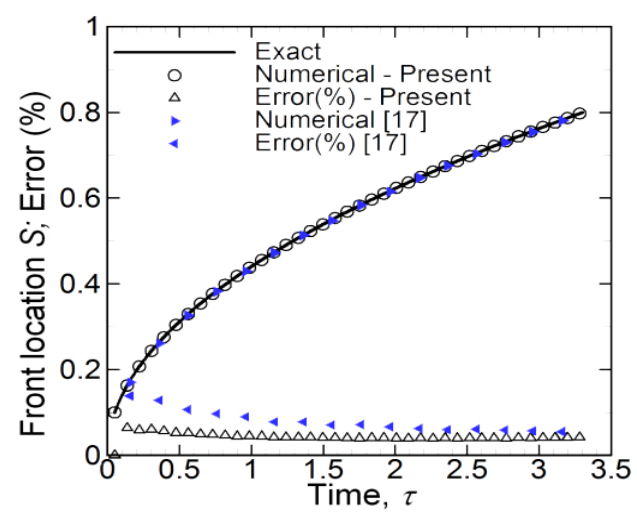

(a) $S t=0.1$

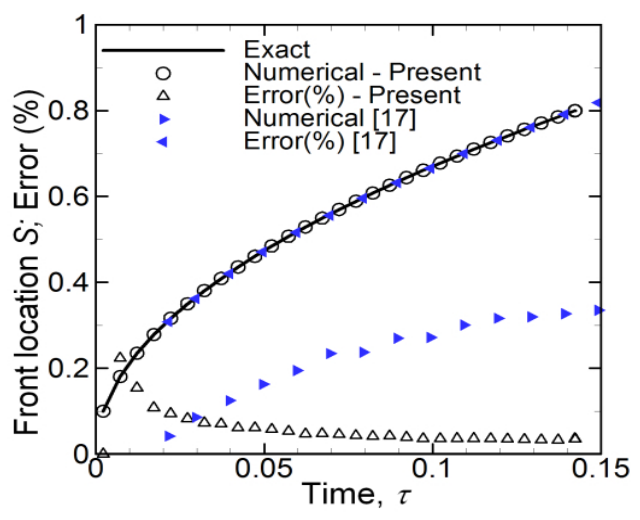

(b) $S t=5$

Fig. 3. Comparisons of the present computational results (open symbols) with the exact solutions (solid line), and with the computational results reported in [17] for

a 1D solidification problem shown in Fig. 2

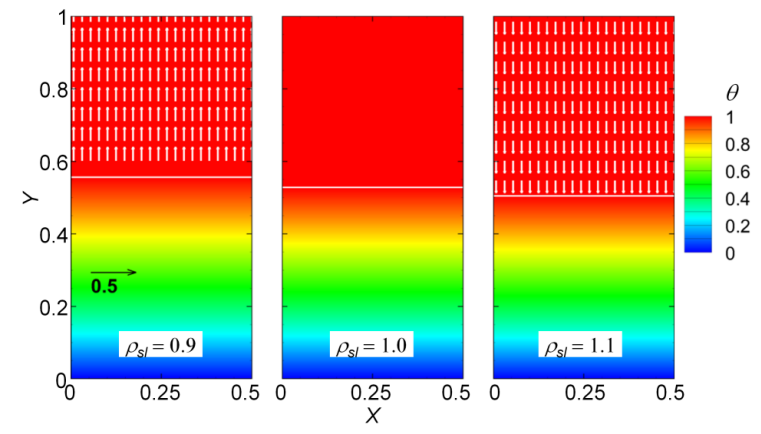

Fig. 4. Flow field during solidification for different solid-to-liquid density ratios $\rho_{s l}$ at $\tau=0.1815$. The velocity is normalized by $\alpha_{l} / H$. The solid line represents the solid-liquid interface.

The Stefan number is 1.0. $\theta$ is the temperature

the liquid velocity vectors midway through solidification for three solid-to-liquid density ratios: $\rho_{s l}=\rho_{s} / \rho_{l}=0.9$ (expansion), $\rho_{s l}=1.0$ (no volume change) and $\rho_{s l}=1.1$ (shrinkage). For $\rho_{s l}=0.9$ (left), the solid has a lower density in comparison with the liquid, and thus the liquid flows away from the solidification front. On the other hand, for $\rho_{s l}=1.1$ (right), the liquid flows toward the solidification front to compensate. For $\rho_{s l}=1.0$ (center), the densities of the solid and liquid phases are equal, resulting in no change in volume, and thus there is no flow in the liquid region. Fig. 4 shows that at this time the solidification front in the case of expansion $\left(\rho_{s l}=0.9\right)$ has advanced further than that in the case of shrinkage $\left(\rho_{s l}=1.1\right)$. In other words, the presence of expansion increases the solidification rate. This result is understandable since the melt flows away from the solid. Fig. 5 confirms that increasing the density ratio leads to a decrease in the solidification rate and thus reduces time to complete solidification. 


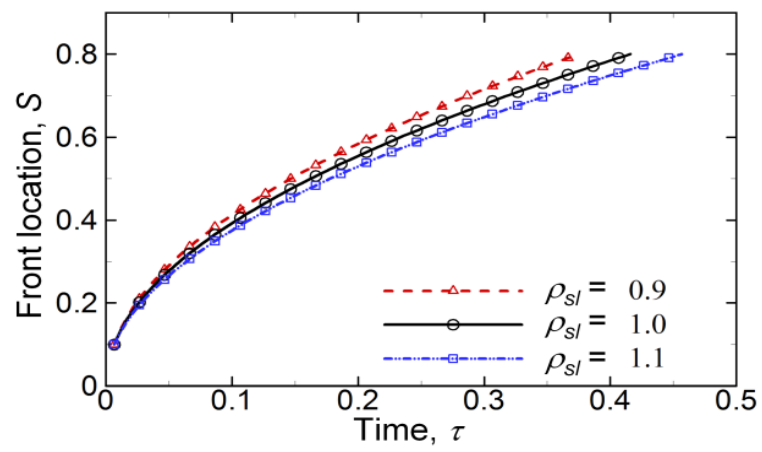

Fig. 5. Temporal variation of the average height of all points on the solidification front for three density ratios shown in Fig. 4

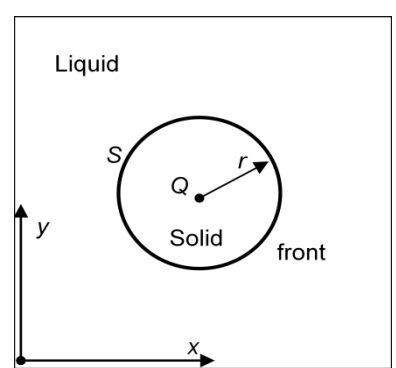

Fig. 6. Two-dimensional Stefan problem

\subsection{Two-dimensional problems}

We next compare our computational results with analytical solutions for a 2D Stefan problem (Fig. 6) in which a line heat source $Q$ causes a circular solid seed at the center to evolve in the direction of increasing the radius of the seed [7]

$$
\frac{\partial\left(C_{p} T\right)}{\partial t}=\frac{1}{r} \frac{\partial}{\partial r}\left(k r \frac{\partial T}{\partial r}\right) .
$$

The exact analytical solution of the temperature field is

$$
T(r, t)=\frac{Q}{4 \pi k_{s}}\left[E i\left(-\frac{r^{2}}{4 \alpha_{s} t}\right)-E i\left(-\lambda^{2}\right)\right],
$$

in the solid, and

$$
T(r, t)=1-E i\left(-\frac{r^{2}}{4 \alpha_{l} t}\right) / E i\left(-\lambda^{2} \alpha_{s} / \alpha_{l}\right),
$$

in the melt. The exact front location is $s(t)=2 \lambda \sqrt{\alpha_{s} t}$, where $\lambda$ is the root of

$$
\frac{Q}{4 \pi} e^{-\lambda^{2}}+\frac{k_{l}}{E i\left(-\lambda^{2} \alpha_{s} / \alpha_{l}\right)} e^{-\lambda^{2} \alpha_{s} / \alpha_{l}}=\frac{\lambda^{2}}{S t} .
$$

Here, $S t=1 /\left(\rho \alpha_{S} L_{h}\right)$ is the Stefan number, $Q$ is the heat source at the center of the circular interface, and $E i$ is the exponential integral function.

Simulations were performed for this $2 \mathrm{D}$ problem with $S t=0.1, Q=40, C_{p s l}=$ $C_{p s} / C_{p l}=0.5$ and $k_{s l}=k_{s} / k_{l}=2$. A $1 \times 1$ domain with a grid resolution of $128 \times 128$ was used. The initial front location and temperature used for simulations were found from the exact solutions at the moment $t=t_{0}$ when a small, circular solid seed with a radius $r=S\left(t_{0}\right)=0.1$ has formed around $(x, y)=(0.5,0.5)$. The temperature conditions at the boundaries for the simulations correspond to the exact solutions at each time step. To circumvent computation of the infinitely negative temperature at $r=0$, i.e. $(x, y)=$ $(0.5,0.5)$, we set the temperature at the four grid points around $(x, y)=(0.5,0.5)$ to the 


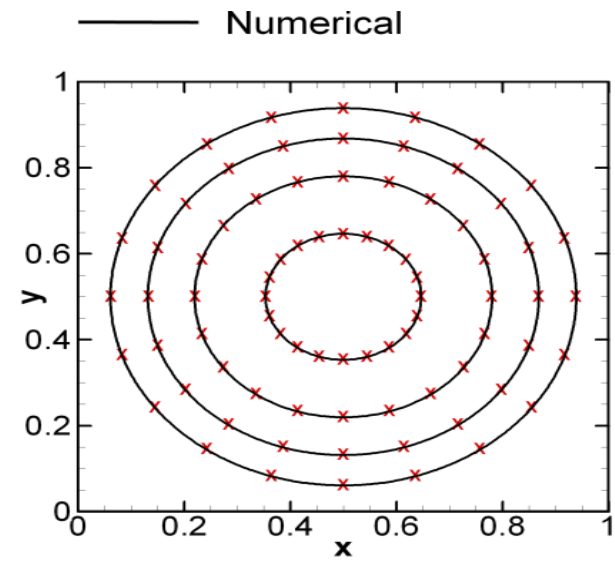

(a) Evolution of the solidfication font

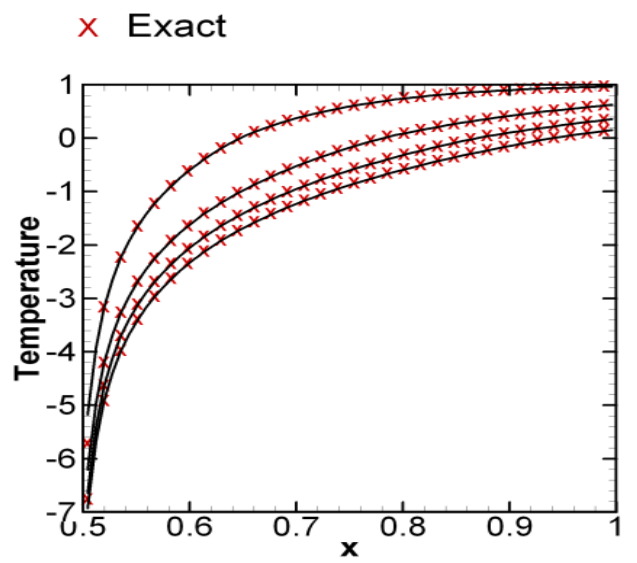

(b) Temperature along $y=0.5$

Fig. 7. Comparisons with exact solutions of Carslaw and Jaeger [7] for a 2D Stefan solidification problem. The data is plotted at time $t=0.03,0.11,0.19$ and 0.27

exact solution. Fig. 7 compares the computational results with the exact solutions for the evolution of the front and temperature. It is clear that the computational results agree excellently with the analytical ones.

\subsection{Ingot casting problem}

The finally considered problem in this study is the solidification process in a rectangular cavity $W \times H$. The cavity is initially filled with liquid at the fusion temperature $T_{m}$. At time $t=0$, a constant temperature $T_{\mathcal{c}}<T_{m}$ is suddenly applied to the side and bottom walls (Fig. 8a). We neglect the effects of natural convection in the liquid region. Accordingly, solidification starts at the walls, and the solid-liquid interface propagates to the center. Pure aluminum is taken to be the phase change material. Its properties can be found in [17]. The nondimensional parameters include

$$
\operatorname{Pr}=\frac{C_{\mathrm{pl}} \mu_{\mathrm{l}}}{k_{1}}=0.0305, S t=\frac{C_{\mathrm{pl}}\left(T_{\mathrm{m}}-T_{c}\right)}{L_{\mathrm{h}}}=0.1, k_{\mathrm{sl}}=\frac{k_{\mathrm{s}}}{k_{1}}=2.5, C_{\mathrm{psl}}=\frac{C_{\mathrm{ps}}}{C_{\mathrm{pl}}}=0.84 .
$$

The temperature is non-dimensionalized as $\theta=\left(T-T_{\mathrm{c}}\right) /\left(T_{m}-T_{\mathrm{c}}\right)$. The nondimensional time is $\tau=t /\left(\rho_{1} C_{\mathrm{pl}} H^{2} / k_{1}\right)$. The density of solid aluminum is different from that of molten aluminum, i.e. $\rho_{s l}=\rho_{s} / \rho_{l}=1.17$. Half of the physical domain is simulated with $H /(0.5 W)=2.0$ and with a grid resolution of $128 \times 256$. Due to suddenly applying the cold temperature at the bottom and side walls, solidification starts at the walls, and the phase change front advances to the center, as shown in Fig. 8b. During the initial stages of solidification, the solidification rate is high because of a large temperature gradient. As solidification proceeds, the solidification rate gradually decreases.

In many works, the authors often neglected the effect of volume change on solidification, i.e $\rho_{s l}$ assumed to be one [17]. However, as previously mentioned, the densities of liquid and solid aluminum are different. This density difference results in volume 


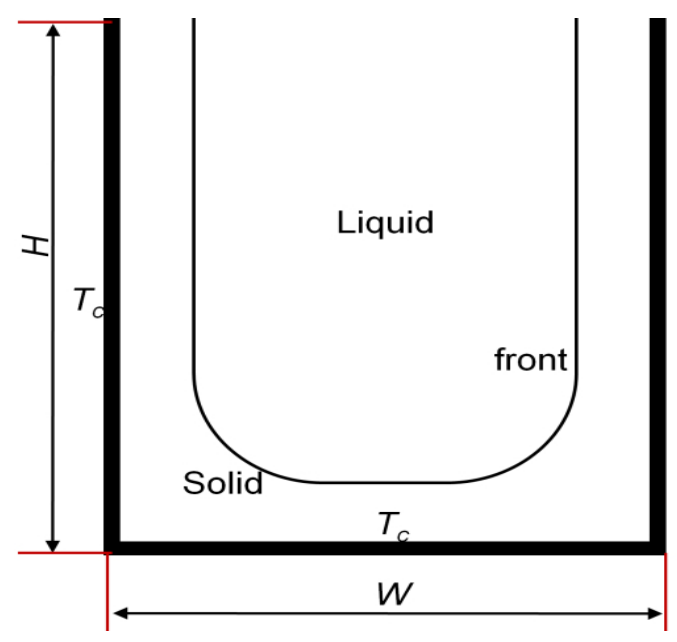

(a)

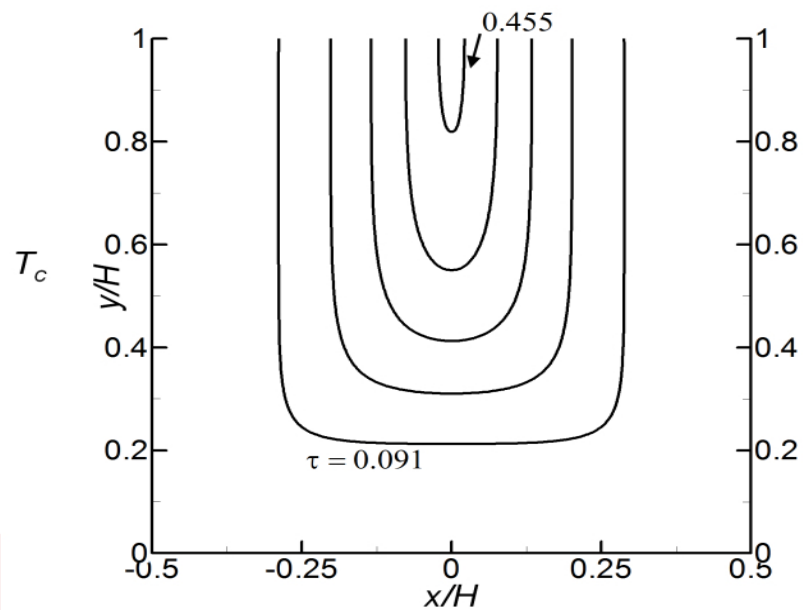

(b)

Fig. 8. Ingot casting problem of aluminum: (a) a schematic and (b) temporal evolution of the solidification front (plotted every $\Delta \tau=0.091$ )

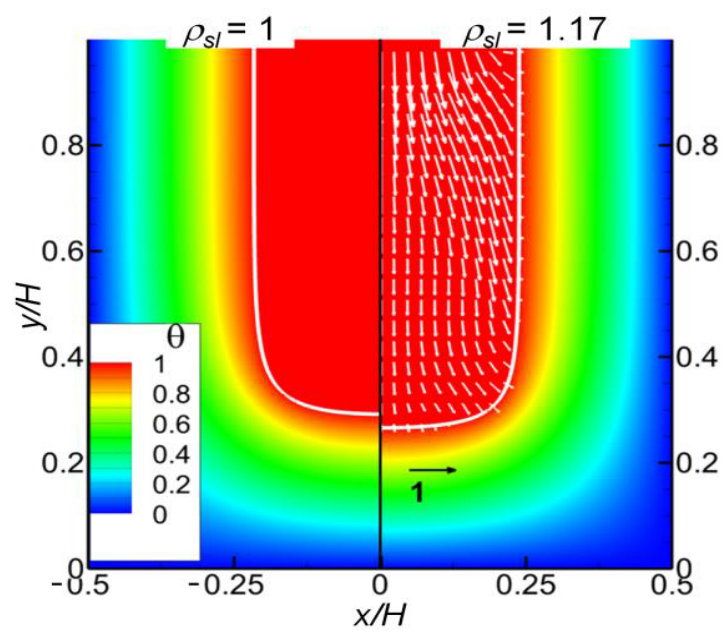

(a)

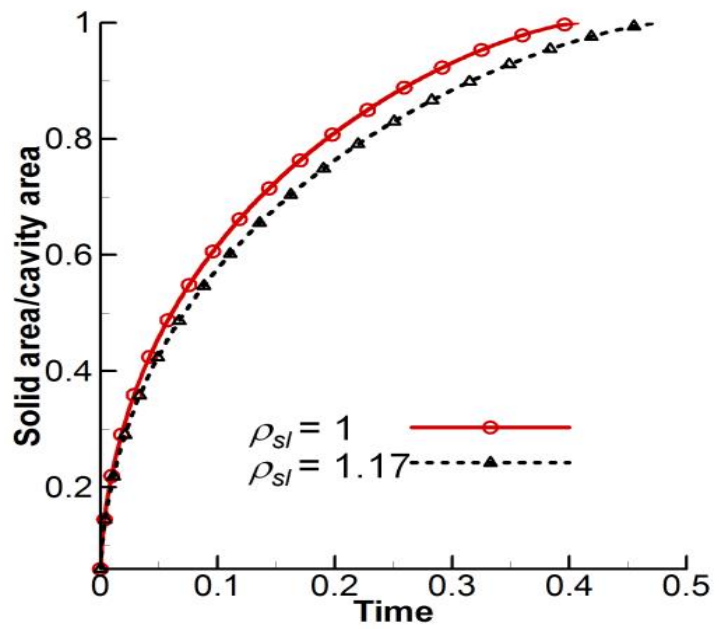

(b)

Fig. 9. Ingot casting problem: (a) flow and temperature field at time $\tau=0.14$, and (b) temporal variation of the ratio of the solid area to the area of the cavity. The solid line in (a) represents the solidification front

change, i.e. shrinkage, upon solidification. To demonstrate this effect, we have also performed a simulation without volume change $\left(\rho_{s l}=1.0\right)$, and compare these two cases, as shown in Fig. 9. As can be ready seen from the figure, density difference between the 
solid and liquid phases causes the liquid to flow toward the solidification front to compensate (Fig. 9a). Accordingly, shrinkage reduces the growth rate and thus increases time to complete solidification as shown in Fig. 9b. This indicates an important role of volume change in the solidification process.

\section{CONCLUSION}

We have presented the front-tracking finite/difference method to simulate solidification of phase change materials in the presence of volume change. The solidification interface is represented by connected points. These front points propagate on the fixed and rectangular grid on which the Navier-Stokes and energy equations are solved. The standard projection method is modified to account for the presence of the solid phase where the velocity is zero. The method is validated through comparisons with exact solutions for one- and two-dimensional solidification problems. These validations are satisfied and support the accuracy of the method. To demonstrate the effects of volume change on the solidification process, computations were performed for the cases without volume change and with volume change. Numerical results indicate that shrinkage, i.e. $\rho_{s l}>1$, reduces the solidification rate while expansion, i.e. $\rho_{s l}<1$, increases the solidification rate.

\section{ACKNOWLEDGMENTS}

This research is funded by Vietnam National Foundation for Science and Technology Development (NAFOSTED) under grant number 107.03-2014.21.

\section{REFERENCES}

[1] A. Faghri and Y. Zhang. Transport phenomena in multiphase systems. Elsevier Academic Press, Burlington, MA, (2006).

[2] C. J. Ho and R. Viskanta. Experimental study of solidification heat transfer in an open rectangular cavity. Journal of Heat Transfer, 105, (3), (1983), pp. 671-673.

[3] G. A. Satunkin. Determination of growth angles, wetting angles, interfacial tensions and capillary constant values of melts. Journal of Crystal Growth, 255, (1), (2003), pp. 170-189.

[4] M. Giangi, T. A. Kowalewski, F. Stella, and E. Leonardi. Natural convection during ice formation: numerical simulation vs. experimental results. Computer Assisted Mechanics and Engineering Sciences, 3, (2000), pp. 321-342.

[5] O. R. Enriquez, A. G. Marin, K. G. Winkels, and J. H. Snoeijer. Freezing singularities in water drops. Physics of Fluids, 24, (9), (2012). Doi: 10.1063/1.4747185.

[6] M. N. Ozisik. Heat conduction. John Wiley \& Sons, (1993).

[7] H. S. Carslaw and J. C. Jaeger. Conduction of heat in solids. Oxford University Press, USA, (1986).

[8] V. R. Voller, C. R. Swaminathan, and B. G. Thomas. Fixed grid techniques for phase change problems: a review. International Journal for Numerical Methods in Engineering, 30, (4), (1990), pp. 875-898.

[9] G. Beckett, J. A. Mackenzie, and M. L. Robertson. A moving mesh finite element method for the solution of two-dimensional stefan problems. Journal of Computational Physics, 168, (2), (2001), pp. 500-518. 
[10] M. Pasandideh-Fard, S. Chandra, and J. Mostaghimi. A three-dimensional model of droplet impact and solidification. International Journal of Heat and Mass Transfer, 45, (11), (2002), pp. 2229-2242.

[11] S. W. J. Welch and J. Wilson. A volume of fluid based method for fluid flows with phase change. Journal of Computational Physics, 160, (2), (2000), pp. 662-682.

[12] Y. Kim, A. Hossain, and Y. Nakamura. Numerical study of melting of a phase change material (PCM) enhanced by deformation of a liquid-gas interface. International Journal of Heat and Mass Transfer, 63, (2013), pp. 101-112.

[13] Y. T. Kim, N. Goldenfeld, and J. Dantzig. Computation of dendritic microstructures using a level set method. Physical Review E, 62, (2), (2000), pp. 2471-2474.

[14] J. B. Collins and H. Levine. Diffuse interface model of diffusion-limited crystal growth. Physical Review B, 31, (9), (1985), pp. 6119-6122.

[15] D. Juric and G. Tryggvason. A front-tracking method for dendritic solidification. Journal of Computational Physics, 123, (1), (1996), pp. 127-148.

[16] N. Al-Rawahi and G. Tryggvason. Numerical simulation of dendritic solidification with convection: two-dimensional geometry. Journal of Computational Physics, 180, (2), (2002), pp. 471496.

[17] C. Y. Li, S. V. Garimella, and J. E. Simpson. Fixed-grid front-tracking algorithm for solidification problems, part I: method and validation. Numerical Heat Transfer, Part B: Fundamentals, 43, (2), (2003), pp. 117-141.

[18] S. Jana, S. Ray, and F. Durst. A numerical method to compute solidification and melting processes. Applied Mathematical Modelling, 31, (1), (2007), pp. 93-119.

[19] N. H. Phan and H. T. Nguyen. Numerical simulation of two-phase free surface flows with a coupling explicit - implicit method. In Proceedings of the 14th Asian Congress of Fluid Mechanics (14 ACFM), Hanoi, Vietnam, (2013). pp. 495-503.

[20] H. D. Phung. Simulation and investigation of interaction between waves and a submerged breakwater. In Proceedings of the 10th Scientific Conference - Institute of Meteorology, Hydrology and Environment, Hanoi, Vietnam, (2007). pp. 211-218.

[21] G. Tryggvason, B. Bunner, A. Esmaeeli, D. Juric, N. Al-Rawahi, W. Tauber, J. Han, S. Nas, and Y. J. Jan. A front-tracking method for the computations of multiphase flow. Journal of Computational Physics, 169, (2), (2001), pp. 708-759.

[22] A. Esmaeeli and G. Tryggvason. Computations of film boiling. Part I: numerical method. International Journal of Heat and Mass Transfer, 47, (25), (2004), pp. 5451-5461.

[23] F. H. Harlow and J. E. Welch. Numerical calculation of time-dependent viscous incompressible flow of fluid with free surface. Physics of Fluids, 8, (12), (1965), pp. 2182-2189. 